\title{
Pregabalin misuse: A silent epidemic
}

A Hapangama, KALA Kuruppuarachchi

\section{Abstract}

Pregabalin is used to treat numerous conditions ranging from peripheral neuropathy to social phobia. Multiple case reports of its abuse potential and dependence, including withdrawal symptoms, have being published in the world literature. Clinicians in Sri Lanka are now encountering patients who are misusing pregabalin due to various reasons, on a regular basis.

SLJ Psychiatry 2019; 10(2): 15-16

\section{Introduction}

Pregabalin has been approved by the US Food and Drug Administration for diabetic peripheral neuropathy, postherpetic neuralgia, fibromyalgia, neuropathic pain associated with spinal cord injury and partial seizures in adults (1). However, it is also used off label for generalized anxiety disorder, panic disorder and social phobia (1). A large number of cases of pregabalin misuse have been reported in European countries and a similar trend seems to be appearing in Sri Lanka (2). The following case histories highlight various presentations of people who were misusing pregabalin.

\section{Case Reports}

A 17-year old school boy presented with worsening of anxiety symptoms, poor sleep, agitation and increased craving for pregabalin. He had been prescribed pregabalin $100 \mathrm{mg}$ a day by a medical practitioner, for social phobia 3 months previously. The patient had started to use more than the prescribed dose and had purchased it without a valid prescription, as he found increasing the dose made him feel "happy and less anxious about others when doing presentations in front of the class". However, recently he had not been able to obtain the same number of capsules.

A 56 year old female on treatment for diabetic neuropathy was referred to the psychiatry clinic as she has had been repeatedly requesting prescriptions for above-therapeutic doses of pregabalin. It was found out that she had been using above-therapeutic doses without a prescription for more than three months, in order to lessen her pain and to feel happier. She reported that she had been commenced on pregabalin $50 \mathrm{mg}$ thrice a day about one year previously, and after about two months the dose had been increased to $100 \mathrm{mg}$ thrice a day on her request. The patient herself had increased the dose during the last few months and on presentation to the psychiatry clinic she was on a total daily dose of $800 \mathrm{mg}$ a day.

A 65-year old woman who was ataxic was found to be using more than the prescribed doses of pregabalin. She had been followed up at a medical clinic for peripheral neuropathy. She had been started on pregabalin $50 \mathrm{mg}$ thrice a day a few months previously, however she had kept increasing the dose herself without medical advice as she felt "she needed more of the medication to relieve the pain". She was found to be taking $600 \mathrm{mg}$ a day of pregabilin. She was not on any other medication and CT scan of her brain was normal.

A 35-year old male, who was in remand prison at the time of referral to the psychiatry clinic, presented with a threeday history of persecutory delusions. It was noted that he had been misusing pregabalin above $500 \mathrm{mg} /$ day for the previous one year. He gave a previous history of heroin use, but denied a history of psychotic symptoms in the past. He stated that his pregabalin or "bud" (as is commonly known in the streets) use was less stigmatized by his family and friends, as he was obtaining it over the counter from a pharmacy for the treatment of 'aches and pains'. His last dose of pregabalin had been on the day prior to him being arrested. 


\section{Discussion}

Pregabalin is a gabapentinoid similar to gabapentin and is a leucine analogue that binds to the alpha 2 delta subunit of voltage-sensitive calcium channels, leading to the closure of $\mathrm{N}$ and $\mathrm{P} / \mathrm{D}$ presynaptic calcium channels. This is reported to reduce excessive neuronal activity and neurotransmitter release (1). It has been described to be structurally similar to gamma amino butyric acid (GABA), but up to now no direct effect of pregabalin has been described on either GABA or its receptors (1).

Pregabalin is reported to be well absorbed after oral administration, with a bioavailability of about $90 \%$ or more (3). It is known to reach peak plasma concentrations within 1.5 days after oral administration, and has a halflife of about 6 hours (3). Pregabalin is reported to achieve a steady state within about $24-48$ hours after repeated administration (3).

The recommended therapeutic dose of pregabalin is 150$600 \mathrm{mg} /$ day (1). Sedation, dizziness, ataxia, diplopia, blurred vision, tremor, confusion, impaired attention, euphoria, asthenia, dry mouth and constipation are some of the side effects of therapeutic doses of pregabalin (1).

An Expert Committee report of the World Health Organization in 2018, warned of tolerance and withdrawal symptoms and behavioral dependence caused by pregabalin (3). Even though pregabalin is not known to directly act at receptor sites associated with drugs of abuse, reports suggest evidence of it producing euphoria, which may explain its potential for abuse (3). It is mentioned that the abuse potential is more in people with a history of substance abuse or psychiatric disorders $(2,4)$.

The routes of abuse of pregabalin include oral, intravenous, nasal insufflation, rectal ("plugging"), smoking and "parachuting" (emptying the content of the capsule into a pouch) (3).

The WHO report describes diaphoresis, tachycardia, hypertension, tremors, diarrhea, anxiety, auditory hallucinations as symptoms of pregabalin withdrawal (3). A case report has described development of psychotic symptoms including persecutory delusions on rapid discontinuation of pregabalin (5).

All four of our patients had been misusing pregabalin, in doses between 300-800 mg a day. After referral they were treated assertively for their underlying conditions (some patients needed to be continued on pregabalin) and are currently being followed up without incident.

We also used behaviour therapy techniques in all four patients to change their respective life-styles.

The misuse of pregabalin in Sri Lanka may increase, as it is a medication that can be legally prescribed, and as certain pharmacists in Sri Lankan tend to dispense medications without a valid prescription. Therefore prescribers must be aware and be vigilant of the high potential for misuse of this drug, which may lead to harmful use or dependence, as well as other complications including death.

\section{Declaration of interest}

None declared

A Hapangama, KALA Kuruppuarachchi, Department of Psychiatry, Faculty of Medicine,University of Kelaniya

Corresponding author: A Hapangama

Email: ahapangama@kln.ac.lk

http://orcid.org/0000-0002-5477-5050

\section{References}

1. Stahl SM. Stahl's essential psychopharmacology: Prescriber's guide. 5th ed. New York: Cambridge University Press; 2017.

2. Gahr M, Freudenmann RW, Hiemke C, Kölle MA, Schönfeldt-Lecuona C. Pregabalin abuse and dependence in Germany: results from a database query. Eur J Clin Pharmacol 2013; 69(6): 1335-42.

3. World Health Organization-Expert Committee on Drug Dependence. Critical review Report: Pregabalin. World Health Organization: Geneva; 2018.

4. Schjerning O, Rosenzweig M, Pottegård A, Damkier P, Nielsen J. Abuse potential of pregabalin: A systematic review. CNS Drugs 2016; 30: 9.

5. Gundogmus I, Karagöz A, Algül A. First-episode psychosis induced by pregabalin withdrawal: a case report, Psych Clin Psychopharmacol 2018; 28(4): 461-63. 